\title{
Array Comparative Genomic Hybridization Analysis Reveals Significantly Enriched Pathways in Canine Oral Melanoma
}

\author{
Ginevra Brocca ${ }^{1 *}$, Serena Ferraresso ${ }^{1}$, Clarissa Zamboni ${ }^{1}$, Elena M. Martinez-Merlo ${ }^{2}$, \\ Silvia Ferro ${ }^{1}$, Michael H. Goldschmidt ${ }^{3}$ and Massimo Castagnaro ${ }^{1}$ \\ ${ }^{1}$ Department of Comparative Biomedicine and Food Science, University of Padua, Legnaro, Italy, ${ }^{2}$ Department of Animal \\ Medicine and Surgery, Complutense University, Madrid, Spain, ${ }^{3}$ School of Veterinary Medicine, University of Pennsylvania, \\ Philadelphia, PA, United States
}

\section{OPEN ACCESS}

Edited by:

Mark W. Dewhirst,

Duke University, United States

Reviewed by:

Jason Somarelli,

Duke University, United States

William Eward,

Duke University, United States

Steven Fiering,

Dartmouth College, United States Miriam Kleiter,

University of Veterinary Medicine Vienna, Austria

*Correspondence:

Ginevra Brocca

ginevra.brocca@gmail.com

Specialty section:

This article was submitted to

Cancer Genetics,

a section of the journal

Frontiers in Oncology

Received: 23 July 2019 Accepted: 26 November 2019 Published: 12 December 2019

Citation:

Brocca G, Ferraresso S, Zamboni C

Martinez-Merlo EM, Ferro $S$, Goldschmidt MH and Castagnaro M (2019) Array Comparative Genomic

Hybridization Analysis Reveals

Significantly Enriched Pathways in

Canine Oral Melanoma.

Front. Oncol. 9:1397.

doi: 10.3389/fonc.2019.01397
Human Mucosal Melanoma (hMM) is an aggressive neoplasm of neuroectodermal origin with distinctive features from the more common cutaneous form of malignant melanoma (CMM). At the molecular level, hMMs are characterized by large chromosomal aberrations rather than single-nucleotide mutations, typically observed in cMM. Given the scarcity of available cases, there have been many attempts to establish a reliable animal model. In pet dogs, Canine Oral Melanoma (COM) is the most common malignant tumor of the oral cavity, sharing clinical and histological aspects with hMM. To improve the knowledge about COM's genomic DNA alterations, in the present work, formalin-fixed, paraffin-embedded (FFPE) samples of COM from different European archives were collected to set up an array Comparative Genomic Hybridization (aCGH) analysis to estimate recurrent Copy Number Aberrations (CNAs). DNA was extracted in parallel from tumor and healthy fractions and 19 specimens were successfully submitted to labeling and competitive hybridization. Data were statistically analyzed through GISTIC2.0 and a pathway-enrichment analysis was performed with ClueGO. Recurrent gained regions were detected, affecting chromosomes CFA 10, 13 and 30, while lost regions involved chromosomes CFA 10, 11, 22, and 30. In particular, CFA 13 showed a whole-chromosome gain in 37\% of the samples, while CFA 22 showed a whole-chromosome loss in 25\%. A distinctive sigmoidal trend was observed in CFA 10 and 30 in 25 and $30 \%$ of the samples, respectively. Comparative analysis revealed that COM and hMM share common chromosomal changes in 32 regions. MAPK- and PI3K-related genes were the most frequently involved, while pathway analysis revealed statistically significant perturbation of cancer-related biological processes such as immune response, drug metabolism, melanocytes homeostasis, and neo-angiogenesis. The latter is a new evidence of a significant involvement of neovascularization-related pathways in COMs and can provide the rationale for future application in anti-cancer targeted therapies.

Keywords: angiogenesis, array comparative genomic hybridization, canine oral melanoma, comparative oncology, copy number aberrations, mucosal melanoma, pathway enrichment analysis 


\section{INTRODUCTION}

Human melanomas of mucosal sites (human Mucosal Melanoma, hMM) are neoplastic diseases of neuroectodermal origin, arising from non-cutaneous melanocytes migrated from the neural crest during embryogenesis (1-4). Although still not fully characterized, hMMs show to rely on numerous copy number changes and whole chromosomes gains or losses, rather than on single-nucleotide mutations, and they lack the typical UV-signature of the cutaneous malignant melanomas (cMM) (4-8). Large chromosomal aberrations, known to be deeply involved in solid tumors development (9), were investigated in hMMs through numerous techniques. Up to date, promising recurrent regions of gains and losses were identified (5) and confirmed by several investigations $(4,7,8)$, in particular amplified portions of HSA $12 \mathrm{q}$ and $5 \mathrm{p}$, which encode for genes as CDK4 and TERT, respectively (7). In addition, CCND1, KIT, and VEGFRA were proposed by a recent review (10) as targets for future investigations. hMMs represent only the $1.3 \%$ of all reported melanomas (1) and they may arise from different sites, as head-and-neck, female genital tract, and anal/rectal mucosa, with a respective 5 years survival rate of $31.7,11.4$, and $19.8 \%$, while cMM has a 5 years survival rate of $80.8 \%$ (1). The highly aggressive biological behavior of hMMs (11) and the scarcity of available cases led to many attempts to establish a reliable animal model for the study of this life-threatening disease. Various in vivo models have been proposed for melanocytic derived-tumors through genetically engineered mice and zebrafish (12). Relevant limitations of these models are the lack of tumor population heterogeneity, combined with the longtime of tumor formation $(12,13)$. Altogether, these studies revealed the necessity of a spontaneous tumor model in non-engineered animals. Among companion animals, equine's primary melanomas have been taken into consideration as a model for hMMs' aberrations (8); however, they showed to have fewer copy number changes compared to hMM, making them a non-fitting model. On the basis of their greater genetic proximity with humans than other models proposed, dogs appear to be a more adequate preclinical surrogate (14). Canine tumors arise spontaneously in an intact immune system, often at a higher rate than in humans, and pet dogs share the same environmental risk factors with the owners. Moreover, dogs have a shorter lifespan and a more rapid neoplastic disease course $(15,16)$. Canine Oral Melanomas (COMs), the most common malignant tumor of the canine oral cavity $(2,17,18)$, are characterized by a clinical evolution and progression, a tendency for local invasion and metastasis (2, 19-22), and a resistance to chemotherapy and radiation therapy $(15,20,23)$, similar to hMM. In 2012, the National Cancer Institute Comparative Melanoma Tumor Board compared histological features of COM and canine melanomas arising in other sites (skin and acral) with hMM and cMM, finding a complete concordance between COMs and hMMs, and suggesting a common enrichment of PI3K and MAPK pathways (13). Given these promising results, the Board strongly encouraged validation of COM as a clinical model for hMM, by deepening the correlation of possible chromosomal, epigenetic and transcriptomic alterations. Molecular studies on COMs detected recurrent gains in CFA 13 and 17, and recurrent losses in CFA 2 and $22(8,24)$. A distinctive sigmoidal trend was also highlighted in CFA 30, with the alternation of gained and lost regions $(8,24)$. Although a large variety of gained and deleted genes was detected, some studies revealed discordant results indicating the need for further investigation on COMs' genetic landscape. In this work, DNA from formalin-fixed, paraffin-embedded (FFPE) samples of COM was collected from two European archives and analyzed through array Comparative Genomic Hybridization (aCGH). This technique takes advantage of the competitive hybridization of matched healthy and pathologic genomic DNA in parallel-extracted from FFPE samples, to estimate recurrent somatic Copy Number Aberrations (CNAs) characteristic of the cluster analyzed.

\section{MATERIALS AND METHODS}

\section{Samples Collection and Selection}

FFPE samples were collected from the archives of the Universities of Padua and Madrid. Initial inclusion criteria for the collection of the samples were a certain diagnosis of COM and sufficient material for nucleic acid extraction. Once collected, one $4 \mu \mathrm{m}$ thick slide was cut from each block and stained with a routine hematoxylin-eosin ( $\mathrm{H} \& \mathrm{E})$ protocol for a second evaluation. To be included in the study, the H\&E slides were reviewed independently by two board-certified veterinary pathologists (American and European) and one expert veterinary pathologist to unequivocally confirm the initial diagnosis of COM, and to assess the presence of an adequate amount of healthy tissue suitable for the nucleic acid extraction. Diagnostic criteria were based upon the guidelines of the World Health Organization (25) and amelanotic specimens were evaluated through anti-Melan-A and anti-PNL2 antibodies. Forty samples were finally evaluated as adequate.

\section{Nucleic Acid Extraction and Purification From FFPE Tissue}

By using H\&E stained slides as a guide, the paraffin blocks were incised in order to separate the tumor bulk from the healthy tissue. Sections $20 \mu \mathrm{m}$-thick were then cut from the blocks using a microtome with disposable blades. Tumor and healthy tissues were then scraped from the slides and put in two different $1.5 \mathrm{ml}$ Eppendorf to be extracted separately. When necessary, more sections were cut in order to provide an adequate amount of healthy tissue material. Care was taken to avoid any possible contamination between tumor and healthy tissue and between different samples, by cleaning microtome, blades, and instruments after processing each specimen. Genomic DNA was extracted using the All-Prep DNA-RNA FFPE KIT (Qiagen ${ }^{\circledR}$ ) according to the manufacturer's instructions, with the use of a heptane solution for deparaffinization steps. Quality and quantity of the extracted DNA were assessed via spectrophotometry with a Nanodrop ND-1000 (Life Technologies ${ }^{\circledR}$ ), while its integrity was checked with an agarose gel electrophoresis, showing a marked degree of degradation in all samples. Only samples with a $\mathrm{A}^{260} / \mathrm{A}^{230}$ ratio of at least 1.5 and a yield of DNA of at least 450 
ng (for both pathological and healthy sections) were admitted to the following steps.

\section{Array Comparative Genomic Hybridization} Genomic DNA from 24 samples was subjected to the cyanine labeling using the SureTag DNA Labeling Kit: DNA extracted from pathological and healthy fractions was labeled independently with Cy 3-deoxyuridine triphosphate (dUTP) and cyanine 5-dUTP, respectively. Cyanine incorporation and final concentration were calculated via spectrophotometry with a Nanodrop ND-1000 and the specific activity was calculated for each sample. Twenty samples, which reached an adequate matched tumor/healthy $\mathrm{Cy} 3$ and $\mathrm{Cy} 5$ specific activity, were then co-hybridized to a 180,000-feature SurePrint G3 Canine CGH Microarray (4-180 K, Agilent Technologies), comprising repeat-masked 60 -mer oligonucleotides distributed at $\sim 2.7 \mathrm{~Kb}$ intervals throughout the dog CanFam2 genome assembly. After $24 \mathrm{~h}$ of incubation at $65^{\circ}$ and $20 \mathrm{rpm}$, arrays were washed following the manufacturer's instruction and scanned at $3 \mu \mathrm{m}$ using an Agilent G2565CA scanner. Image data were processed using Feature Extraction version 11.5, and Genomic Workbench version 7.0.

\section{CNAs Analysis}

Data were filtered to exclude probes exhibiting non-uniform hybridization or signal saturation and were normalized using the centralization algorithm with a threshold of eight and fuzzy ON. The ADM-2 algorithm was applied to define CNAs using a "three probes minimum" filter. Only autosomes were analyzed. The Cy5/Cy3 intensity ratios for each spot were converted into log2 ratios. Aberrant chromosome intervals were selected by using Agilent Genomic Workbench v. 7.0. A copy number gain was defined as a $\log 2$ ratio $>0.25$ and a copy number loss was defined as a $\log 2$ ratio $<-0.25$. Chromosomal locations were defined in terms of their Megabase $(\mathrm{Mb})$ position. To identify significant CNAs the Genomic Identification of Significant Targets in Cancer (GISTIC2.0) (26) algorithm was also applied, as implemented in CGHtools software. The GISTIC2.0 module identifies regions of the genome that are significantly amplified or deleted across samples. Each aberration is assigned a G-score that considers the amplitude as well as the frequency of its occurrence across samples. False Discovery Rate q-values are then calculated for the aberrant regions, and regions with q-values below a userdefined threshold are considered significant. Log2ratios $\geq 0.25$ and $\leq-0.25$ were assigned as the threshold for gain and loss detection, while amplification and deletion were defined as having a $\log 2$ ratio $\geq 1$ and $\leq-1$. False Discovery Rate (FDR) $\leq 0.05$ was set as the limit of significance.

\section{Comparison Between Canine and Human CNAs}

To compare the canine CNA profile with aberrations already described in the recent human literature, orthologous regions were identified using the Liftover Batch Coordinate Conversion Tool (http://genome.ucsc.edu/cgi-bin/hgLiftOver), as already done in previous studies $(8,24)$. In summary, the genome coordinates of the 180.00060 -mer probes of each array were mapped firstly to the canine reference genome CanFam3.1, and then to the human reference genome GRCh38/hg38. The syntenic human regions were then compared with published data $(5,7,8,24)$, to detect those regions shared by both hMMs and COMs. A comparative analysis between data produced herein, and recently published studies regarding the detection of CNAs in the canine genome through several techniques (as WES, WGS, aCGH, and FISH) $(8,24,27,28)$, was also performed.

\section{Pathway Enrichment Analysis}

Orthologous human genes were identified using the Ensemble Genome Browser (http://www.ensembl.org/index.html) and four lists of genes were employed for pathway analysis: (i) Gains with penetrance $\geq 25 \%$ (GR25), (ii) Gains with penetrance $\geq 40 \%$ (GR40), (iii) Losses with penetrance $\geq 25 \%$ (LR25) and (iv) regions highlighted as significant by Gistic analysis (GS). Genes were analyzed as human orthologs using the ClueGo plugin (29) for the software Cytoscape 3.7.1, an open-source Java tool that extracts the non-redundant biological information for large clusters of genes. In ClueGO, the kappa score is used to define term-term interrelations (edges), and functional groups based on shared genes between terms. Here, Homo sapiens was used as the control organism, and genes were uploaded as human orthologs named by the SymbolID. The genes were assigned to a network based on the updated ontologies: KEGG, GO Biological process, GO Immuno, REACTOME, and WIKIPATHWAYS. The significance of each term was calculated with a standard hypergeometric two-sided test. Networks were created on the basis of a kappa score threshold of 0.5 and a minimum of 3 genes in every network forming at least $10 \%$ of the total associated genes in each particular network, as previously done (28). Pathways' $P$-values were adjusted with BenjaminiHochberg and the "fusion" option was also applied to reduce the redundancy. Pathways were then represented taking advantage of Cytoscape's complex visualization environment, as kappa scorebased functional groups, and named by the most significant term of each group.

\section{Immunohistochemistry and Immunohistochemical Assessment}

For each of the 20 samples analyzed through the aCGH technique, a $4 \mu \mathrm{m}$-thick section was cut and mounted on a polarized glass slide (Superfrost ${ }^{\circledR}$ Plus, Thermo Scientific ${ }^{\circledR}$ ), and tested with the mouse monoclonal antibody Ki67 (Dako ${ }^{\circledR}$ ) diluted 1:50. Immunohistochemistry was performed with an automatic immunostainer (Ventana Benchmark GX, Roche-Diagnostic) using an ultraView universal alkaline phosphatase RED detection kit (Ventana Medical System Inc.), which provides a red chromogen reaction, and hematoxylin counterstain. The use of the red chromogen allowed avoiding bleaching reactions in pigmented COMs, in which $\mathrm{DAB}$ chromogen is often unusable, preserving the integrity of antigens. A Ki67 index was established for each sample on the base of the methodology described by Bergin et al. (30), which showed to be prognostic with a cutoff of 19.5 average cells per high power field (hpf). 


\section{Data Access}

The data discussed in this publication have been deposited in NCBI's Gene Expression Omnibus (31), and are accessible through GEO Series accession number GSE131923 (https://www. ncbi.nlm.nih.gov/geo/query/acc.cgi?acc $=\sim$ GSE131923).

\section{RESULTS}

\section{Collected Samples and Immunohistochemical Analysis}

A total of 20 samples, inclusive of the tumor and matched normal tissue, were selected for cyanine labeling and showed both an adequate yield and an adequate specific activity to be further subjected to the aCGH analysis. Only one case showed poor quality of hybridization and was excluded from this study, bringing the number of samples to 19. For each sample, an IHC with the Ki67 antibody was successfully performed, and the Ki67 index was established. Based on the study conducted by Bergin et al. (30), we established a threshold Ki67 value of $>19.5$ for the prediction of death (or euthanasia) due to melanoma by 1 year post-diagnosis. Based on the Ki67 value, samples were then classified as with a GOOD $(\mathrm{G})$ or BAD (B) possible prognosis. The G group included 5/20 samples, and the B group 15/20 (including the one excluded from the aCGH cohort). Samples and available clinical data of dogs from which they were collected are listed in Table S1 in Supplementary Materials.

\section{Genomic Pattern of Aberration}

CNA analysis allowed the identification of both focal and broad (near the size of a chromosome arm) chromosomal aberrations, distinguished in gains and losses (Figure 1). Two samples (A5, A35) did not present any aberration, while in the remaining ones, the mean number of aberrations per sample was 27.6 (range: 271). The pattern of genomic aberrations was evaluated for gained and lost regions with a penetrance $\geq 25 \%$ and consisted of 53 gained regions, with size ranging from $12.7 \mathrm{~Kb}$ to $30.9 \mathrm{Mb}$ (with a mean length of $0.7 \mathrm{Mb}$ ), and 20 lost regions ranging from $60 \mathrm{bp}$ to $40.5 \mathrm{Mb}$ (mean length of $2 \mathrm{Mb}$ ).

The most frequently gained regions (penetrance $\geq 25 \%$ ) affected chromosomes CFA 10,13, and 30, while lost regions involved most frequently chromosomes CFA 10, 11, 22, and 30. Among the regions with gains, 8 showed a penetrance $\geq 40 \%$, with regions chr30:17522685-17773010 and chr30:1784767418058012 showing 45\% penetrance. Among regions with losses, nine had a penetrance $\geq 30 \%$ and the most frequent loss was chr11:41248370-41248429, with a 35\% penetrance. CNAs with penetrance $\geq 25 \%$ and corresponding genes are listed in Table S2 in Supplementary Materials. Chromosomes that appeared to be more affected by gains and losses were CFA 13 and 22 . CFA 13 showed a whole-chromosome gain in the $37 \%(7 / 19)$ of the samples, while CFA 22 showed a whole-chromosome loss in the $25 \%(5 / 19)$ of the samples, with the loss of region 0.2 to $54 \mathrm{Mb}$ that reached a $30 \%$ penetrance. Additionally, a recurrent and distinctive alternation of gained and lost regions (sigmoidal trend) was observed on CFA 10 (25\% of the samples, $5 / 19)$ and 30 (30\% of the samples, 6/19). No aberrations significantly associated with a Ki67 index greater or lower than 19.5 were identified. The most frequent aberration observed was the loss of region chr11:41248370-41248429 in the G group, recurrent in three out of five samples. Microarray data were then interrogated using the GISTIC2.0 algorithm, to identify CNAs with a statistically significant frequency. A total of 20 significant gained regions were located on CFA 9, 10, 13, and 30. Those regions and the corresponding genes (reported in Table S3 in Supplementary Materials) were mostly overlapping

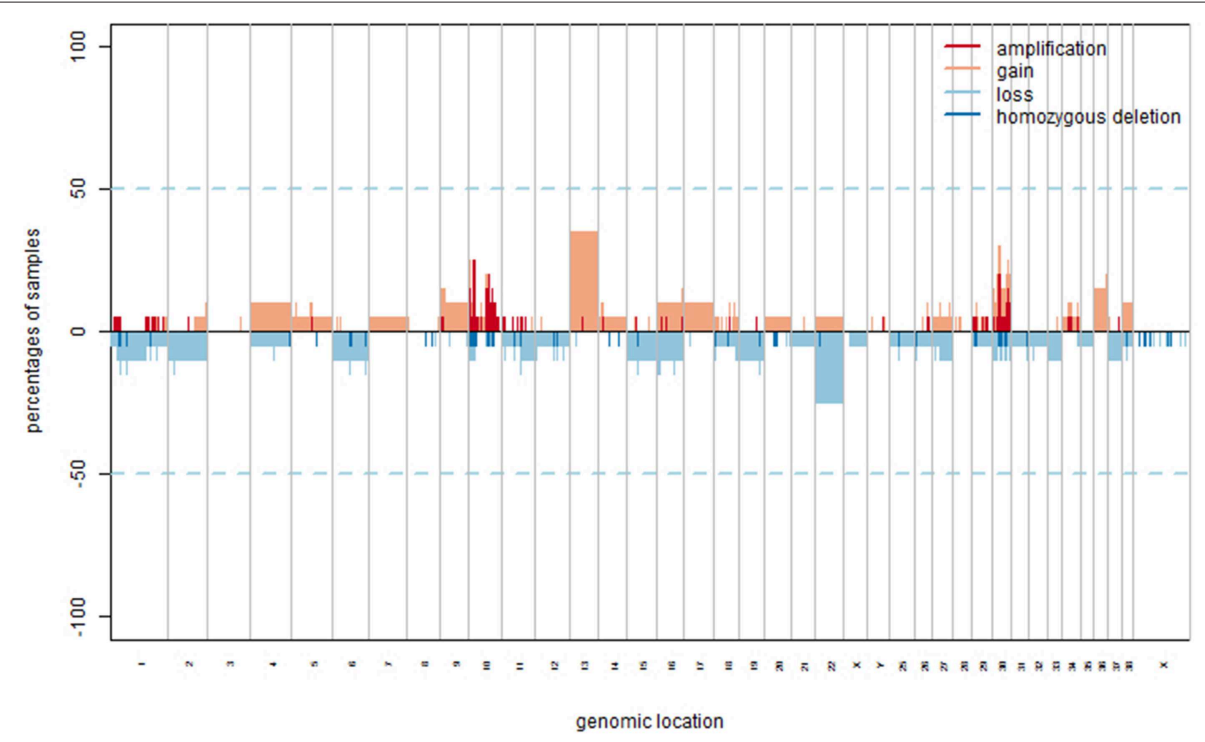

FIGURE 1 | CNAs in COM. Copy number gains and amplification are indicated in orange and red, respectively representing a log 2 ratio $\geq 0.25$ and $\geq 1$. Copy number losses and deletion are indicated in light and dark blue, respectively representing a $\log _{2}$ ratio $\leq-0.25$ and $\leq-1$. 


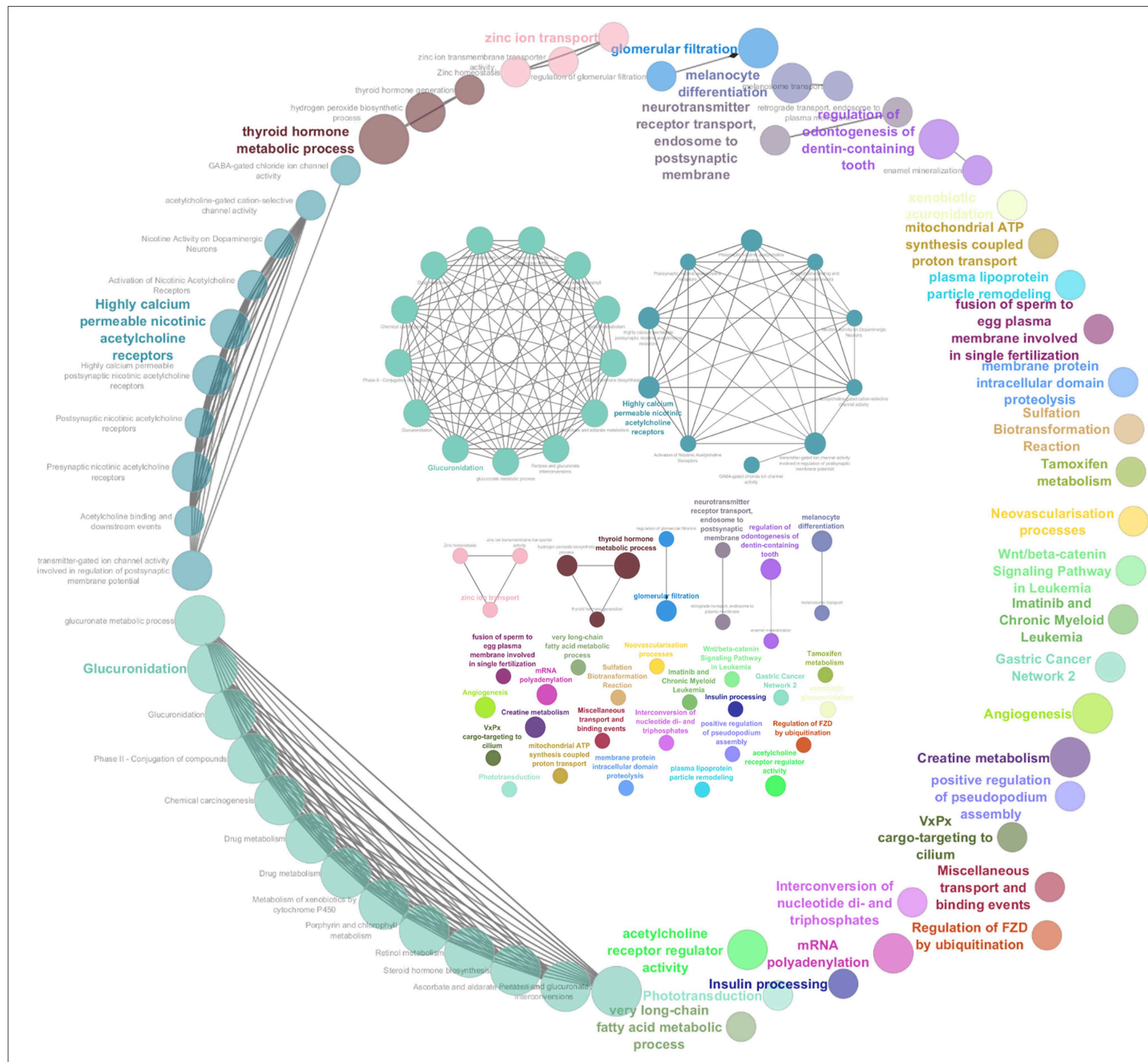

FIGURE 2 | Graphic representation of the results obtained from the pathway enrichment analysis. These pathways were obtained subjecting the genes extrapolated from gained regions with a penetrance $\geq 25 \%$ (GR25 list) to ClueGO. They are represented all together in a circular shape as functional groups, which are visible in more detail inside the circle.

with those showing higher penetrance across samples. The regions' size ranged from $33.9 \mathrm{~Kb}$ to $52.3 \mathrm{Mb}$, with a mean length of $4.2 \mathrm{Mb}$. CFA 10 and CFA 30 were affected by significant amplification in $36.8 \%(7 / 19)$ and $26.3 \%$ (5/19) of the samples, respectively. The three most frequent minimum common regions (MCRs) of CFA 10 were 1.7 to $1.9 \mathrm{Mb}, 10.9$ to $11.8 \mathrm{Mb}$ and 43.6 to $45 \mathrm{Mb}$, while the most frequent MCRs of CFA 30 were 13.6 to $13.9 \mathrm{Mb}$, and 16.2 to $17.9 \mathrm{Mb}$. The GISTIC2.0 algorithm failed to identify statistically significant lost regions. A hierarchical clustering technique aimed to identify molecular features potentially correlated with the Ki67 index showed inconsistent results.

\section{Pathway Enrichment Analysis}

To generate a summary of the pathways likely involved in the tumorigenesis of COMs, four separate lists (i.e., GR25, GR40, LR25, and GS, see Methods) were submitted to the ClueGO tool to identify significantly enriched pathways. Pathways were considered significant if having an adjusted Benjamini-Hochberg $P<0.05$. The enrichment analysis identified 60 significant pathways for the group GR25 (Figure 2), 10 significant pathways for the group LR25, and 49 significant pathways for the group GS. No pathways were found significantly enriched when analyzing the GR40 group. The complete list of significant pathways and genes is reported in Table S4 in Supplementary Materials, while 
TABLE 1 | List of part of the significantly enriched pathways.

\begin{tabular}{|c|c|c|c|}
\hline Pathways & cPValue & Associated genes found & Source \\
\hline Angiogenesis & $<0.01$ & ANGPT1, KDR, PDGFRA, PTK2 & GR25, GS \\
\hline Glucuronidation & $<0.01$ & $\begin{array}{l}\text { UGT2A1, UGT2A3, UGT2B10, UGT2B11, UGT2B15, UGT2B17, } \\
\text { UGT2B28, UGT2B4, UGT2B7 }\end{array}$ & GR25, GS \\
\hline Highly calcium permeable nicotinic acetylcholine receptors & $<0.01$ & CHRNA3, CHRNA5, CHRNB4 & GR25, GS \\
\hline Wnt/beta-catenin Signaling Pathway in Leukemia & 0.03 & FZD6, MYC, PYGO1, WIF1 & GR25, GS \\
\hline Tamoxifen metabolism & 0.02 & SULT1E1, UGT2B15, UGT2B7 & GR25, GS \\
\hline Imatinib and Chronic Myeloid Leukemia & 0.02 & KIT, MYC, PDGFRA & GR25, GS \\
\hline Gastric Cancer Network 2 & 0.01 & ATAD2, DSCC1, FAM91A1, MYC & GR25, GS \\
\hline Chemical carcinogenesis & $<0.01$ & $\begin{array}{l}\text { MGST3, UGT2A1, UGT2A3, UGT2B10, UGT2B11, UGT2B15, } \\
\text { UGT2B17, } \\
\text { UGT2B28, UGT2B4, UGT2B7 }\end{array}$ & GR25, GS \\
\hline T-helper 1 type immune response & $<0.01$ & IL18R1, IL18RAP, IL1RL1, SOCS5, TRAPPC9, UTP3 & GS \\
\hline Hippo-Yap signaling & 0.01 & MAP4K4, NDRG1, STK3 & GS \\
\hline $\begin{array}{l}\text { Negative regulation of cell migration involved in sprouting } \\
\text { angiogenesis }\end{array}$ & $<0.01$ & DLL4, SPRED1, THBS1 & LR25 \\
\hline Hydrolysis of LPC & $<0.01$ & JMJD7-PLA2G4B, PLA2G4B, PLA2G4D, PLA2G4E & LR25 \\
\hline
\end{tabular}

CPValue, P-value corrected with Benjamini-Hochberg; GR25, gains with penetrance $\geq 25 \%$; LR25, Iosses with penetrance $\geq 25 \%$; GS, regions highlighted as significant by Gistic analysis.

the most interesting, together with corresponding genes, are listed in Table 1. Pathways found enriched in the GR25 group included Angiogenesis $(P<0.01)$, Glucuronidation $(P<0.01)$, Highly calcium permeable nicotinic acetylcholine receptors $(P<$ $0.01)$, and Wnt/beta-catenin Signaling Pathway in Leukemia $(P=$ $0.03)$. Interestingly, many pathways related to Drug metabolism $(P<0.01)$ were also found significantly enriched (see Table 1$).$ Other significant pathways were Gastric Cancer Network 2 ( $P=$ $0.01)$, Chemical carcinogenesis $(P<0.01)$, and Insulin processing $(P=0.01)$. Most of the other pathways found to be enriched were melanocytes-related, such as Melanocyte differentiation $(P$ $<0.01$ ), or linked to the dental apparatus, e.g., Regulation of odontogenesis of dentin-containing tooth $(P<0.01)$. Noteworthy, in the GR25 group and GS group, enriched pathways were mostly overlapping. Additionally, GS enriched pathways included the T-helper 1 type immune response $(P<0.01)$ and Hippo-Yap signaling pathways $(P=0.01)$. Regarding the LR25 group, the most enriched pathways were Negative regulation of cell migration involved in sprouting angiogenesis $(P<0.01)$, and Hydrolysis of LPC $(P<0.01)$.

\section{Comparative Analysis Between Canine and Human CNAs}

Orthologous chromosomal regions in canine and human genome were examined to assess conserved CNAs between COMs and hMMs. In human literature, frequent gains of regions of human chromosomes HSA 1, 4, 5, 6, 7, 8, 11, 12, 17, 20, and losses of regions of HSA 3, 4, 6, 8, 9, 10, 11, 17, 21, have been reported $(5,7)$. The comparative analysis revealed 32 regions shared between COMs and hMMs, and regarded gains on CFA
9, 10, and 13, orthologous to HSA 17, 12, and 8-4 (respectively), and losses on CFA 11, 22 and 30, orthologous to HSA 9, 13, and 15 (respectively). A representation is visible in Tables $2 \mathrm{~A}-\mathbf{C}$, while more details about the syntenic human regions are given in Table 3. In particular, the region characterized by the greatest recurrence was CFA 13:1722286-32543593, corresponding to HSA 8:99748261-136957380, already reported $(5,8,24)$, and detected with a penetrance of $35 \%$ in this work. Regarding genes proposed as candidates for the tumorigenesis by other studies, a further concordance between COMs and hMMs was observed with the detection from gained regions of MYC, KIT and, for the first time, PDGFRA, although the latter was found gained only in the $12.5 \%$ of the hMMs analyzed (6) (Table 4A). A concordance can also be found in the loss of regions coding for BUB1B, KNSTRN, CYSLTR2, and SPRED1 (8) (Table 4B). Interestingly, also B2M was found imbalanced in both COMs and hMMs (8), but affected by a gain in the present work, instead of a loss. Other frequently reported events in hMMs are usually gains of $\operatorname{BRAF}(6,8,24), \operatorname{MDM} 2(6,8), \mathrm{CDK} 4$ $(5-7,24)$, and CCND1 $(5,6,24)$; losses of CDKN2A, PTEN $(5,6,24)$, and TP53 $(4,6)$. However, aberrations involving the latter candidate genes were not found in the present study. A new promising target gene was also absent: PTPRJ was found lost in both hMMs and COMs (8), and was reported as inactivated by somatic mutations in COMs (28), but was not involved in the present cohort.

\section{Comparison With Published Canine CNAs}

Recently, several studies focused on COM's genetic landscape. The most frequently reported aberrations are gains on CFA 13, 17, and losses on CFA 11, 15, 
TABLE 2 | Representation of the aberrated regions detected in the present study (CanFam3.1 annotation), showing a correspondent canine or human syntenic region in other studies, which are indicated through the bibliographic number: Wong et al. (8), Poorman et al. (24), Giannuzzi et al. (27), Hendricks et al. (28), Hayward et al. (6), Curtin et al. (5), Lyu et al. (7), and Furney et al. (4). CNAs are listed on the base of the chromosomal location, and divided into 3 groups: (A) gained regions with a penetrance $\geq 25 \%$ (GR25); (B) lost regions with a penetrance $\geq 25 \%$ (LR25); (C) represents gained regions found statistically significant by Gistic analysis (GS).

\begin{tabular}{|c|c|c|c|c|c|c|c|c|c|c|c|}
\hline \multirow[b]{2}{*}{ CHR } & \multirow[b]{2}{*}{ Present study } & \multicolumn{4}{|c|}{ CANINE studies } & \multicolumn{6}{|c|}{ HUMAN studies } \\
\hline & & $\begin{array}{l}\text { Wong } \\
\text { et al. (8) }\end{array}$ & $\begin{array}{l}\text { Poorman } \\
\text { et al. } \\
\text { (24) }\end{array}$ & $\begin{array}{l}\text { Giannuzzi } \\
\text { et al. } \\
\text { (27) }\end{array}$ & $\begin{array}{l}\text { Hendricks } \\
\text { et al. } \\
\text { (28) }\end{array}$ & $\begin{array}{l}\text { Wong } \\
\text { et al. (8) }\end{array}$ & $\begin{array}{l}\text { Poorman } \\
\text { et al. } \\
\text { (24) }\end{array}$ & $\begin{array}{l}\text { Hayward } \\
\text { et al. (6) }\end{array}$ & $\begin{array}{l}\text { Curtin } \\
\text { et al. (5) }\end{array}$ & $\begin{array}{l}\text { Lyu } \\
\text { et al. (7) }\end{array}$ & $\begin{array}{l}\text { Furney } \\
\text { et al. (4) }\end{array}$ \\
\hline
\end{tabular}

\section{(A) GR25}

10

7814333-7827061

7827061-7896552

7896552-8860169

8860169-8889136

8889136-9197582

9225870-9593296

9593296-9724425

9724425-9752955

$13 \quad 1722286-32543593$

32543593-34917864

34917864-60030824*

60030824-60110727

$30 \quad$ 16069762:16221290

16221290:16599297

16599298:16724522

16724522:16973676

16973676:17914975

\section{(B) LR25}

11

38095457-38199500

38199500-38219624

38219624-38219683

38219683-38263483

38263483-38372087

\begin{tabular}{ll}
\hline 22 & $\frac{102563-10473893}{1305764-2530849}$ \\
\cline { 2 - 2 } 30 & $\frac{2530849-2552538}{2552538-2770691}$ \\
$\frac{2670691-4634866}{5314049-9109654}$
\end{tabular}

(C) GS

9 1839468-1890597

2038907-2071775

$10 \quad 6204314-9752896$

9948113-10047999

$13 \quad 101-23829651$

23906345-60168042*

(24)

HUMAN studies 


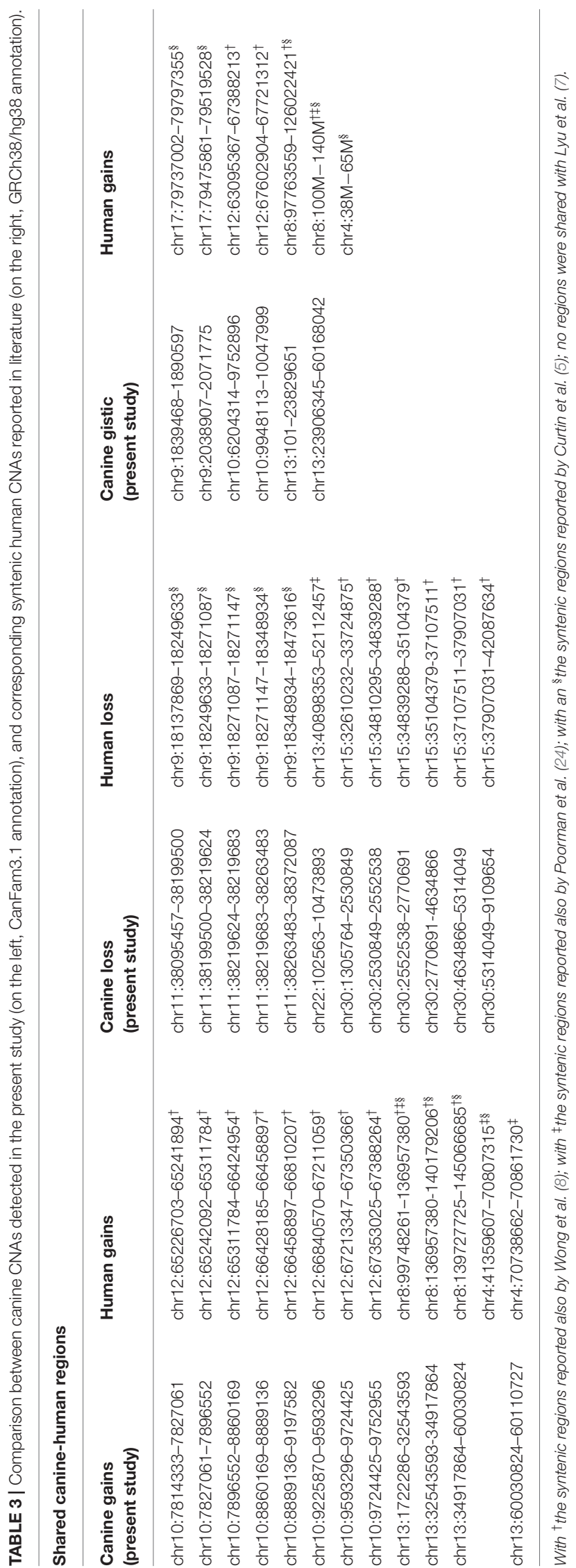

studies $(24,28)$, and had a penetrance $\geq 30 \%$ and $\geq 25 \%$ in this work, respectively.

Several genes which have been indicated to play a significant role in the development of COMs and hMMs were involved in CNAs, comprising gains of TRPM7 $(24,27)$, MYC $(8,24)$, KIT (24, 28), WIF1 (8), SLC27A2, GABPB1, USP8, SPPL2A, CYP19A1 (27) (Table 4A) and losses of RB1 $(8,24)$, LCP1, BUB1B, KNSTRN, CYSLTR2 (8), SPRED1 $(24,27)$, and FAM98B (27) (Table 4B). On the contrary, other genes such as MDM2 $(27,28)$ or CDK4 $(8,28)$, were not confirmed by our study. Finally, it is noteworthy that other genes involved in CNAs such as ADAM10, and genes belonging to gene families SNORA, SNORD, SLC25A, RPL, and RBM, have been recently shown to be highly expressed in metastatic COMs (32).

More details about the target genes taken into consideration (from both canine and human studies) are graphically represented in Tables 4A,B.

\section{DISCUSSION}

In the present study, more than 250 FFPE samples have been collected from archive material. However, due to the stringent inclusion criteria aimed to analyze only samples with a sufficient amount of paired healthy DNA, only 40 were considered adequate candidates for the aCGH analysis. The low number of samples has been a limitation, and a likely cause of the inconsistent results obtained from the hierarchical clustering, with only $5 / 20$ cases with a Ki67 value $<19.5$. Since the presence of healthy tissue was a major restriction in the recruitment of cases, to ease future analysis the inclusion of a portion of presumed healthy tissue in the diagnostic sample is therefore recommended. The highly homogeneous cohort of samples obtained and the matching DNA for each sample allowed to overcome all potential discrepancies deriving from the use of genomic dog pools, which could have led to false correlations with race, age, sex, and health conditions.

A 50\% increase in the DNA extraction yield was obtained by using the heptane as deparaffinization agent instead of the more toxic xylene (33). Precipitation of the extracted DNA with ethanol allowed to obtain a good DNA quality in some of the samples with an initial poor $\mathrm{A}^{260} / \mathrm{A}^{230}$ ratio.

Chromosomal aberrations detected in this study partially overlap with those already documented in other works on canine species, and the software analysis showed both new and known enriched pathways. Only the GR40 group failed to identify significantly enriched pathways, probably due to the limited number of genes included in the list.

The most characteristic aberration is confirmed to be the sigmoidal pattern of CFA 10 and CFA30 $(8,24,28)$. However, the biological significance of these recurrently lost-gained regions is still unclear. Future studies correlating the presence of lost-gained regions and clinical data could improve our understanding of this specific molecular feature of COM.

MDM2 $(8,27,28), \operatorname{CDK} 4(8,28)$, and $\operatorname{CDKN} 2 \mathrm{~A}(24,27$, 28 ), which were found altered by other authors, were not identified as significantly aberrated by GISTIC algorithm in 
TABLE 4 | Comparison of the target genes found gained (A) or lost (B) in this and other studies, which are indicated through the bibliographic number: Wong et al. (8), Poorman et al. (24), Giannuzzi et al. (27), Hendricks et al. (28), Hayward et al. (6), Curtin et al. (5), Lyu et al. (7), and Furney et al. (4).

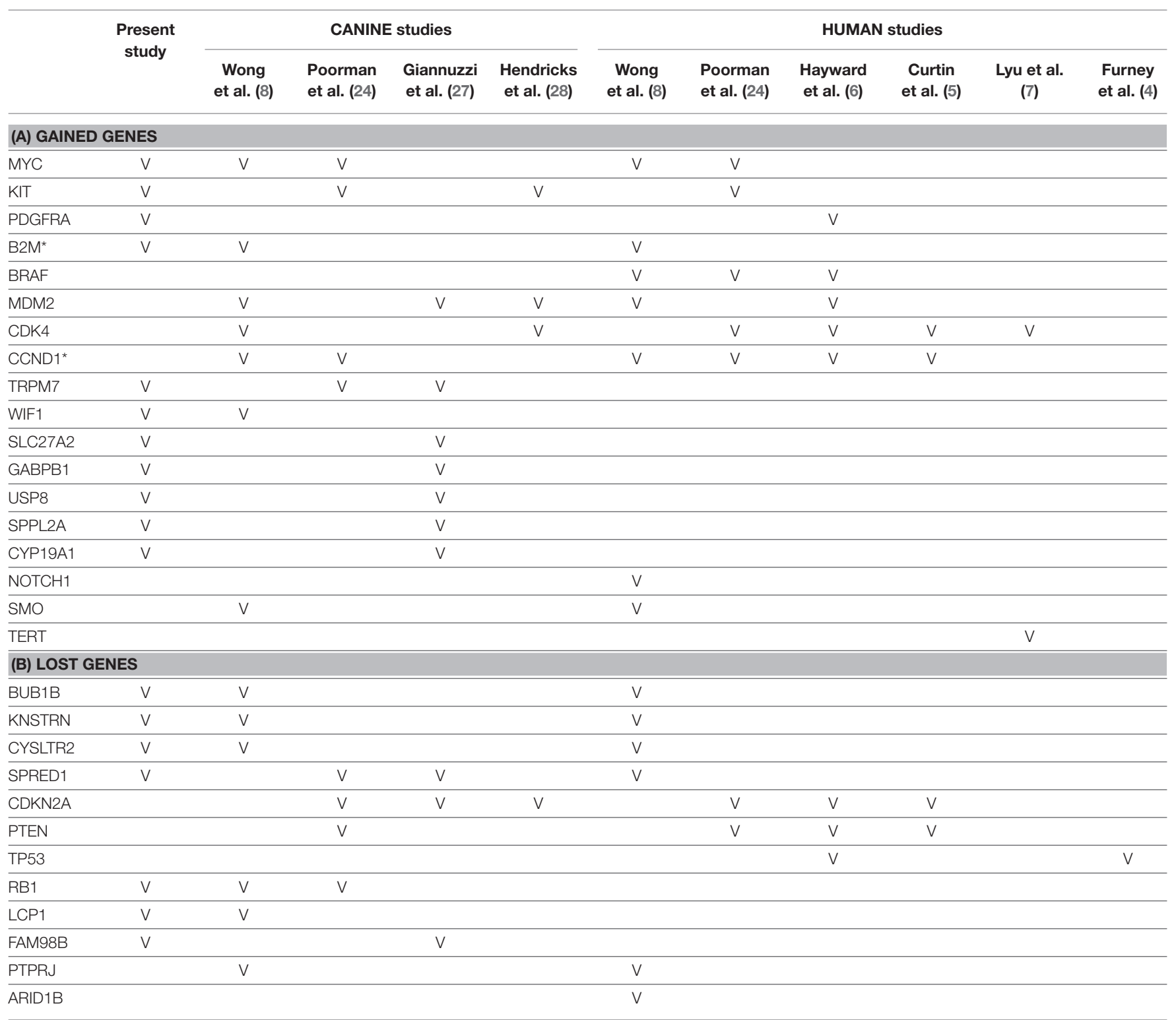

With * the genes B2M and CCND1: B2M was found gained in the present study, but was reported as lost by Wong et al. (8); CCND1 was reported as gained in hMMs, but lost in COMs, by Wong et al. (8) and Poorman et al. (24).

this study. As reported also in hMM (4), MDM2 is known to favor tumor formation by acting on the tumor suppressor gene p53 (34). Although the reason for this discrepancy is not known, a significant gain of the MDM2 binding protein (MTBP) was instead present in our samples. These data, together with a copy number gain involving the p53 binding protein (TP53BP1), confirm the dysregulation of p53 family members in COMs. Moreover, a significant enrichment of the Hippo-Yap signaling pathway, which is strongly and sometimes contradictorily intertwined with the p53 pathway (35), was also detected.

CDK4 and its inhibitor CDKN2A are main actors of the cell cycle proliferation since they regulate Cyclin D1, allowing or not the transition from G1 to $\mathrm{S}$ phase. Although a direct involvement of CCND1 has been identified only in human acral and mucosal melanomas (5), and not in COMs (24), high expression of Cyclin D1 protein in COMs has been recently documented (36). Furthermore, a gain of the coding gene for Cyclin B2 (CCNB2), and a copy number imbalance of Eukaryotic Translation Initiation Factor family members (EIF2C2, EIF2AK4, EIF3E, EIF3H, EIF3J), were detected. Interestingly, the member EIF4E of the family is reported to increase the level of Cyclin D1 protein in vitro $(37,38)$, while other members (components of the EIF3 complex, in particular), have been correlated to human cancer (39-49), and human melanoma $(41,50,51)$. 
Another notable aberration is the loss of the tumor suppressor gene Retinoblastoma 1 (RB1), considered the governor of the cell cycle. RB1 has been already associated with other human and canine cancer types and it is strictly correlated to the cyclins' family. Even if with a mechanism different from those proposed in other works, cyclins activation appears then highly involved also in our cohort of COMs.

Among the genes related to cell proliferation and mitosis, GRHL2, a transcription factor able to bind the promoter region of TERT, was found to be gained. Although TERT is one of the most frequently involved genes in human non UV-induced melanomas $(7,10)$ it has never been found amplified in COMs. However, the gain of GRHL2 suggests that TERT expression may play a role in COMs.

A loss of mitosis-related genes was also highlighted. Loss of KNSTRN, required for correct chromosome segregation, BUB1, a checkpoint for mitosis progression, and TACC3, a stabilizer of the mitotic spindle, were detected.

COM is known to share with hMM the activation of MAPK and PI3K pathways, showing also similar responses to targeted therapies $(52,53)$. Aberrations are recognized to be part of the activation mechanism, and many MAPK-related genes are encoded by CFA 30 (orthologous to HSA 15) (8, 24, 28). Genes responsible for MAPK and PI3K activation found in this work comprise RASGRP1, MYC, FGF7, ANGPT1, TRPM7 (gained), and SPRED1 (lost). As reported in other works $(6,24)$, an imbalance of a wide variety of genes coding for tyrosine kinases receptors was also detected. These genes are abnormally activated in a wide range of human and animal tumors, inducing uncontrolled tumor proliferation (54). Our data showed an imbalance of the proto-oncogene c-KIT, and of other kinases such as PTK2, STK3, TEC, PDGFRA, VEGFR2, and CD63. It is noteworthy that VEGF receptors are involved in metastatic behavior in human melanoma studies (55), while PDGF receptors' expression has been shown to bear prognostic significance in COMs (22). Finally, CD63, a wellknown melanoma-associated antigen (also called "MelanomaAssociated Antigen ME491"), has a role in VEGFA signaling.

As reported by other authors (28), the involvement of $\mathrm{PI} 3 \mathrm{~K} / \mathrm{mTOR} / \mathrm{AKT}$ signaling pathway is also suggested by the significant enrichment of the Insulin processing pathway, which is well-known to activate PI3K. The latter is also regulated by DEPTOR and MAP2K1 genes, which have been found altered in our study. Noteworthy, PI3K and mTOR are essential for the maintenance of the stem cell status in neural progenitor cells (56), and their abnormal involvement appears strategical for the tumorigenesis of neural crest-derived tumors such as COM.

Interestingly, the Angiogenesis pathway was enriched in both GR25 and GS lists (derived from the gained regions), and its suppressive pathway (Negative regulation of cell migration involved in sprouting angiogenesis) was enriched in LR25 list (derived from the lost regions). In addition, other vascularrelated pathways, such as Neovascularization processes, were found enriched. Vessels proliferation, which plays an important role in several types of human melanomas (57), appears then to be deeply implicated in COMs' pathogenesis, and likely in their metastatic behavior. Neoangiogenesis is an obligatory phenotypic step for the establishment of distant metastases throughout the body: without an angiogenic process, the freshly-proliferated cells would incur into hypoxia and lack of nutrients, making the microenvironment adverse for the metastases survival (57). The main genes involved in endothelial and vascular cell proliferation, and reported by the ClueGo analysis, were ANGPT1, VEGFR2, PDGFRA, PTK2 for both GR25 and GS groups, and DLL4, SPRED1, THBS1, which are involved in the negative regulation of vessel sprouting, for LR25. Additionally, an imbalance (gains and losses) on the endothelin receptor type B gene (EDNRB), which role is crucial for melanocytes development (3) and for vessel homeostasis, was also detected. Interestingly, antibody-drug conjugates (ADC) targeting the endothelin $\mathrm{B}$ receptor $\left(\mathrm{ET}_{\mathrm{B}} \mathrm{R}\right)$ both in vitro and in vivo systems $(58,59)$, show anti-tumor activity (even if partial) in hMMs (59). Given these premises, further evaluation of $\mathrm{ET}_{\mathrm{B}} \mathrm{R}^{\text {'s expression in }}$ COMs could be promising to evaluate the application of ADC therapies also in dogs.

Furthermore, our data show the involvement of many other genes closely related to an angiogenic phenotype. Many genes coding for interleukin receptors, inflammatory molecules that contribute to neovascularization through endothelial cells migration and proliferation, and through metalloproteinases overexpression (as MMP19), were found gained in this study. Significantly, in hMM, a close relationship between IL-8, its receptor CXCR2, and MMP-2, is well-established (60, 61). Additional involved genes were those coding for fibroblast growth factors (FGF7 and FGF14), for lipid lysophosphatidic acids (LPAR6) - which are known to contribute to angiogenesis and lymphangiogenesis (57) - and for melanoma proliferationand migration-related properties such as ZIC5 (62).

The GISTIC2.0 algorithm indicated the T-helper 1 type immune response pathway was significantly enriched (genes are listed in Table 1). Although in dogs therapeutic trials based on immunomodulation did not reach consistent results (63-65), these data strengthen the view of melanoma as a promising target for immunogenic therapies (66).

A general loss of many components of the T-cell homeostasis such as LCP1, TNFSF11, LRCH1, TRIM13 (which acts together with MDM2), RASGRP1, and GPR18 was found. On the contrary, the gain of PDCD7, involved in glucocorticoid-induced apoptosis in mouse T-cells (67), was detected.

Pathways related to drug metabolism were also found to be enriched, with Glucuronidation being the most significant. The cause is attributable to the high representation of the UGT-family genes, which are involved in the activity of the enzyme glucuronosyltransferase and phase II metabolism, and constitute an important pathway for xenobiotic elimination from the organism. Although the involvement of Glucuronidation in COM's behavior is still unclear, its potential role should be taken into consideration for future clinical trials and drug testing.

Not surprisingly, genes involved in the pathogenesis of other tumors were found to be altered. Significant gains of genes ATAD2, DSCC1, FAM91A1, and MYC brought to the enrichment of the Gastric Cancer Network 2 pathway, and ATAD2 is a cancer-associated protein which can also induce the expression of Cyclin D1 and MYC (68). 
Another enriched pathway, which has been frequently associated with cancer, was the Wnt/beta-catenin Signaling Pathway in Leukemia, which mediates the cell transduction signal. Gains of genes such as FZD6, PYGO1, and WIF1, causing Wnt inhibition, and MYC, were found. The involvement of Wnt signaling has been associated to numerous types of cancer, as glioblastoma (69), esophageal (70), ovarian (71), breast (72), colorectal (73), prostate (74), and lung (75) cancers and also to cutaneous melanomas (76). Gains of Wnt inhibitory genes in COM may still have a role in the complex deregulation of the Wnt pathway, which leads to carcinogenesis. However, further studies are needed to clarify this point.

The enrichment of pathways related to melanocytes' development (from neural stem cells) and pigmentation have been already reported in hMMs (7). In accordance, a significant enrichment of the Highly calcium permeable nicotinic acetylcholine receptors, and of the Melanocyte differentiation pathways were found in our study. Finally, the Regulation of odontogenesis of dentin-containing tooth pathway was also significantly enriched. Genes involved in the enrichment of this last pathway, namely AMTN, ENAM, RSPO2, and TNFRSF11B, encode, respectively, for the ameloblast protein amelotin, teeth component enamelin (a Wnt activator), and a TNF receptor. The involvement of these genes may be related to the frequent tendency of COMs to affect the oral cavity, and gingiva in particular (77). The application of aCGH on 19 COMs contributed to increase our knowledge of genetic aberrations in this canine tumor. We confirmed aberrational patterns noted also by other authors, as the sigmoidal trend in CFA 10 and 30. Thirty-two regions here detected showed to be syntenic with hMM-related regions and confirmed a common involvement of MAPK and PI3K pathways in COMs and hMMs. Moreover, our data suggest a strong involvement in COM's tumorigenesis of neovascularizationrelated pathways. These new data, together with the encouraging evidence of anti-angiogenic factors target therapies in human melanomas, remark the role of the dog as a model for hMMs and encourage new studies aimed to test the application of anti-angiogenic factors in the treatment of advanced and/or metastatic COMs.

\section{DATA AVAILABILITY STATEMENT}

The datasets generated for this study can be found in the NCBI's Gene Expression Omnibus, accession number: GSE131923.

\section{REFERENCES}

1. Chang AE, Karnell LH, Menck HR. The National Cancer Data Base report on cutaneous and noncutaneous melanoma. Cancer. (1998) 83:1664-78. doi: 10. 1002/(SICI)1097-0142(19981015)83:8<1664::AID-CNCR23>3.0.CO;2-G

2. Smith SH, Goldschmidt $\mathrm{MH}$, McManus PM. A comparative review of melanocytic neoplasms. Vet Pathol. (2002) 39:651-78. doi: 10.1354/vp.39-6-651

3. Dupin E, Le Douarin NM. Development of melanocyte precursors from the vertebrate neural crest. Oncogene. (2003) 22:3016-23. doi: 10.1038/sj.onc. 1206460

\section{ETHICS STATEMENT}

Ethical review and approval was not required for the animal study because only formalin-fixed, paraffin-embedded surgical biopsies from canine tumors were used in the present work. Tissues were provided by the archives of the Department of Comparative Biomedicine and Food Science (University of Padova) and of the Department of Animal Medicine and Surgery (Complutense University). Written informed consent for participation was not obtained from the owners because the biopsies collected from the archives had already been approved for research purposes by the attending veterinarians, at the moment they sent the material to our facilities.

\section{AUTHOR CONTRIBUTIONS}

SeF and MC conceptualized the study design. MC acquired the funds and supervised the whole project. CZ and EM-M collected and recruited the cases and their available information. GB and $\mathrm{CZ}$ performed the IHC analysis when necessary. SiF, MG, and MC reviewed H\&E and IHC slides to confirm the diagnosis. GB and $\mathrm{SeF}$ performed the aCGH analysis. GB curated the processed data and wrote the original draft. All authors reviewed and approved the final manuscript.

\section{FUNDING}

This present work was financed with internal resources of the University of Padua.

\section{ACKNOWLEDGMENTS}

The authors thank Dr. Annamaria Di Meglio and Dr. Stefano Capomaccio, for the helpful support in data processing. The authors also thank the Journal of Comparative Pathology Educational Trust, which supported the submission of some preliminary data of this work to the 2019 ESVP-ECVP Congress.

\section{SUPPLEMENTARY MATERIAL}

The Supplementary Material for this article can be found online at: https://www.frontiersin.org/articles/10.3389/fonc. 2019.01397/full\#supplementary-material

4. Furney SJ, Turajlic S, Stamp G, Nohadani M, Carlisle A, Thomas JM, et al. Genome sequencing of mucosal melanomas reveals that they are driven by distinct mechanisms from cutaneous melanoma. J Pathol. (2013) 230:261-9. doi: $10.1002 /$ path.4204

5. Curtin JA, Fridlyand J, Kageshita T, Patel HN, Busam KJ, Kutzner $\mathrm{H}$, et al. Distinct sets of genetic alterations in melanoma. $N$ Engl J Med. (2005) 353:2135-47. doi: 10.1056/NEJMoa 050092

6. Hayward NK, Wilmott JS, Waddell N, Johansson PA, Field MA, Nones K, et al. Whole-genome landscapes of major melanoma subtypes. Nature. (2017) 545:175-80. doi: 10.1038/nature22071 
7. Lyu J, Song Z, Chen J, Shepard MJ, Song H, Ren G, et al. Wholeexome sequencing of oral mucosal melanoma reveals mutational profile and therapeutic targets. J Pathol. (2018) 244:358-66. doi: 10.1002/path.5017

8. Wong K, van der Weyden L, Schott CR, Foote A, Constantino-Casas F, Smith $\mathrm{S}$, et al. Cross-species genomic landscape comparison of human mucosal melanoma with canine oral and equine melanoma. Nat Commun. (2019) 10:353. doi: 10.1038/s41467-018-08081-1

9. Albertson DG, Collins C, McCormick F, Gray JW. Chromosome aberrations in solid tumors. Nat Genet. (2003) 34:369-76. doi: 10.1038/ng1215

10. Merkel EA, Gerami P. Malignant melanoma of sun-protected sites: a review of clinical, histological, and molecular features. Lab Invest. (2017) 97:630-5. doi: 10.1038/labinvest.2016.147

11. Carvajal RD, Spencer SA, Lydiatt W. Mucosal melanoma: a clinically and biologically unique disease entity. J Natl Compr Cancer Netw. (2012) 10:34556. doi: 10.6004/jnccn.2012.0034

12. van der Weyden L, Patton EE, Wood GA, Foote AK, Brenn T, Arends MJ, et al. Cross-species models of human melanoma. J Pathol. (2016) 238:152-65. doi: $10.1002 /$ path.4632

13. Simpson RM, Bastian BC, Michael HT, Webster JD, Prasad ML, Conway $\mathrm{CM}$, et al. Sporadic naturally occurring melanoma in dogs as a preclinical model for human melanoma. Pigment Cell Melanoma Res. (2014) 27:37-47. doi: $10.1111 / \mathrm{pcmr} .12185$

14. Lindblad-Toh K, Wade CM, Mikkelsen TS, Karlsson EK, Jaffe DB, Kamal M, et al. Genome sequence, comparative analysis and haplotype structure of the domestic dog. Nature. (2005) 438:803-19. doi: 10.1038/nature04338

15. Vail DM, MacEwen EG. Spontaneously occurring tumors of companion animals as models for human cancer. Cancer Invest. (2000) 18:781-92. doi: 10.3109/07357900009012210

16. Paoloni M, Khanna C. Translation of new cancer treatments from pet dogs to humans. Nat Rev Cancer. (2008) 8:147-56. doi: 10.1038/nrc2273

17. Todoroff RJ, Brodey RS. Oral and pharyngeal neoplasia in the dog: a retrospective survey of 361 cases. J Am Vet Med Assoc. (1979) 175:567-71.

18. Goldschmidt MH. Benign and malignant melanocytic neoplasms of domestic animals. Am J Dermatopathol. (1985) 7:203-12. doi: 10.1097/00000372-198501001-00039

19. Ramos-Vara JA, Beissenherz ME, Miller MA, Johnson GC, Pace LW, Fard A, et al. Retrospective study of 338 canine oral melanomas with clinical, histologic, and immunohistochemical review of 129 cases. Vet Pathol. (2000) 37:597-608. doi: 10.1354/vp.37-6-597

20. Patrick RJ, Fenske NA, Messina JL. Primary mucosal melanoma. J Am Acad Dermatol. (2007) 56:828-34. doi: 10.1016/j.jaad.2006.06.017

21. Gillard M, Cadieu E, De Brito C, Abadie J, Vergier B, Devauchelle P, et al. Naturally occurring melanomas in dogs as models for non-UV pathways of human melanomas. Pigment Cell Melanoma Res. (2014) 27:90-102. doi: $10.1111 / \mathrm{pcmr} .12170$

22. Iussich S, Maniscalco L, Di Sciuva A, Iotti B, Morello E, Martano $\mathrm{M}$, et al. PDGFRs expression in dogs affected by malignant oral melanomas: correlation with prognosis. Vet Comp Oncol. (2017) 15:462-9. doi: $10.1111 /$ vco. 12190

23. Bergman PJ, Wolchok JD. Of Mice and Men (and Dogs): development of a xenogeneic DNA vaccine for canine oral malignant melanoma. Cancer Ther. (2008) 6:817-26.

24. Poorman K, Borst L, Moroff S, Roy S, Labelle P, Motsinger-Reif A, et al. Comparative cytogenetic characterization of primary canine melanocytic lesions using array CGH and fluorescence in situ hybridization. Chromosome Res. (2015) 23:171-86. doi: 10.1007/s10577-014-9444-6

25. Goldschmidt MH, Dunstan RW, Stannard AA, von Tscharner C, Walder EJ, Yager JA. Histological Classification of Epithelial and Melanocytic Tumors of the Skin of Domestic Animals. Washington, DC: Armed Forces Institute of Pathology in cooperation with the American Registry of Pathology and TheWorld Health Organization Colaborating Center for Worldwide Reference on Comparative Oncology (1998). p. 38-40.

26. Mermel CH, Schumacher SE, Hill B, Meyerson ML, Beroukhim R, Getz G. GISTIC2.0 facilitates sensitive and confident localization of the targets of focal somatic copy-number alteration in human cancers. Genome Biol. (2011) 12:R41. doi: 10.1186/gb-2011-12-4-r41

27. Giannuzzi D, Marconato L, Elgendy R, Ferraresso S, Scarselli E, Fariselli $\mathrm{P}$, et al. Longitudinal transcriptomic and genetic landscape of radiotherapy response in canine melanoma. Vet Comp Oncol. (2019) 17:308-16. doi: $10.1111 /$ vco. 12473

28. Hendricks WPD, Zismann V, Sivaprakasam K, Legendre C, Poorman $\mathrm{K}$, Tembe $\mathrm{W}$, et al. Somatic inactivating PTPRJ mutations and dysregulated pathways identified in canine malignant melanoma by integrated comparative genomic analysis. PLoS Genet. (2018) 14:e1007589. doi: 10.1371/journal.pgen.1007589

29. Bindea G, Mlecnik B, Hackl H, Charoentong P, Tosolini M, Kirilovsky A, et al. ClueGO : a Cytoscape plug-in to decipher functionally grouped gene ontology and pathway annotation networks. Bioinformatics. (2009) 25:1091-3. doi: 10.1093/bioinformatics/btp101

30. Bergin IL, Smedley RC, Esplin DG, Spangler WL, Kiupel M. Prognostic evaluation of Ki67 threshold value in canine oral melanoma. Vet Pathol. (2011) 48:41-53. doi: 10.1177/0300985810388947

31. Edgar R, Domrachev M, Lash AE. Gene Expression Omnibus: NCBI gene expression and hybridization array data repository. Nucleic Acids Res. (2002) 30:207-10. doi: 10.1093/nar/30.1.207

32. Bowlt Blacklock KL, Birand Z, Selmic LE, Nelissen P, Murphy S, Blackwood L, et al. Genome-wide analysis of canine oral malignant melanoma metastasis-associated gene expression. Sci Rep. (2019) 9:6511. doi: 10.1038/s41598-019-42839-x

33. Stockert JC, López-Arias B, Del Castillo P, Romero A, Blázquez-Castro A Replacing xylene with $\mathrm{n}$-heptane for paraffin embedding. Biotech Histochem. (2012) 87:464-7. doi: 10.3109/10520295.2012.701764

34. Forslund A, Zeng Z, Qin LX, Rosenberg S, Ndubuisi M, Pincas H, et al. MDM2 gene amplification is correlated to tumor progression but not to the presence of SNP309 or TP53 mutational status in primary colorectal cancers. Mol Cancer Res. (2008) 6:205-11. doi: 10.1158/1541-7786.MCR07-0239

35. Furth N, Aylon Y, Oren M. p53 shades of Hippo. Cell Death Differ. (2018) 25:81-92. doi: $10.1038 /$ cdd.2017.163

36. Zamboni C, Brocca G, Ferraresso S, Ferro S, Sammarco A, Dal Corso C, et al. Cyclin D1 immunohistochemical expression and somatic mutations in canine oral melanoma. Vet Comp Oncol. (2019) 1-8:12539. doi: 10.1111/vco.12539

37. Rosenwald IB, Lazaris-Karatzas A, Sonenberg N, Schmidt EV. Elevated levels of cyclin D1 protein in response to increased expression of eukaryotic initiation factor 4E. Mol Cell Biol. (1993) 13:7358-63. doi: 10.1128/MCB.13.12.7358

38. Rosenwald IB, Kaspar R, Rousseau D, Gehrke L, Leboulch P, Chen JJ, et al. Eukaryotic translation initiation factor $4 \mathrm{E}$ regulates expression of cyclin D1 at transcriptional and post-transcriptional levels. J Biol Chem. (1995) 270:2117680. doi: 10.1074/jbc.270.36.21176

39. Avdulov S, Li S, Michalek V, Burrichter D, Peterson M, Perlman DM, et al. Activation of translation complex eIF4F is essential for the genesis and maintenance of the malignant phenotype in human mammary epithelial cells. Cancer Cell. (2004) 5:553-63. doi: 10.1016/j.ccr.2004. 05.024

40. Lin L, Holbro T, Alonso G, Gerosa D, Burger MM. Molecular interaction between human tumor marker protein p150, the largest subunit of elF3, and intermediate filament protein K7. J Cell Biochem. (2001) 80:483-90. doi: 10.1002/1097-4644(20010315)80:4\%3C483::aid-jcb1002\%3E3.0.co;2-b

41. Gillis LD, Lewis SM. Decreased eIF3e/Int6 expression causes epithelial-tomesenchymal transition in breast epithelial cells. Oncogene. (2013) 32:3598605. doi: 10.1038/onc.2012.371

42. Shi J, Kahle A, Hershey JW, Honchak BM, Warneke JA, Leong SP, et al. Decreased expression of eukaryotic initiation factor $3 \mathrm{f}$ deregulates translation and apoptosis in tumor cells. Oncogene. (2006) 25:4923-36. doi: 10.1038/sj.onc.1209495

43. Pincheira R, Chen Q, Zhang JT. Identification of a $170-\mathrm{kDa}$ protein over-expressed in lung cancers. Br J Cancer. (2001) 84:1520-7. doi: 10.1054/bjoc.2001.1828

44. Rothe M, Ko Y, Albers P, Wernert N. Eukaryotic initiation factor 3 p110 mRNA is overexpressed in testicular seminomas. Am J Pathol. (2000) 157:1597-604. doi: 10.1016/S0002-9440(10)64797-9

45. Nupponen NN, Porkka K, Kakkola L, Tanner M, Persson K, Borg A, et al. Amplification and overexpression of p40 subunit of eukaryotic translation initiation factor 3 in breast and prostate cancer. Am J Pathol. (1999) 154:177783. doi: 10.1016/S0002-9440(10)65433-8 
46. Miyazaki S, Imatani A, Ballard L, Marchetti A, Buttitta F, Albertsen H, et al. The chromosome location of the human homolog of the mouse mammary tumor-associated gene INT6 and its status in human breast carcinomas. Genomics. (1997) 46:155-8. doi: 10.1006/geno.1997.4996

47. Doldan A, Chandramouli A, Shanas R, Bhattacharyya A, Cunningham JT, Nelson MA, et al. Loss of the eukaryotic initiation factor $3 \mathrm{f}$ in pancreatic cancer. Mol Carcinog. (2008) 47:235-44. doi: 10.1002/mc.20379

48. Li Z, Lin S, Jiang T, Wang J, Lu H, Tang H, et al. Overexpression of eIF3e is correlated with colon tumor development and poor prognosis. Int J Clin Exp Pathol. (2014) 7:6462-74.

49. Sesen J, Cammas A, Scotland SJ, Elefterion B, Lemarié A, Millevoi S, et al. Int6/eIF3e is essential for proliferation and survival of human glioblastoma cells. Int J Mol Sci. (2014) 15:2172-90. doi: 10.3390/ijms15022172

50. Doldan A, Chandramouli A, Shanas R, Bhattacharyya A, Leong SP, Nelson $\mathrm{MA}$, et al. Loss of the eukaryotic initiation factor $3 \mathrm{f}$ in melanoma. $\mathrm{Mol}$ Carcinog. (2008) 47:806-13. doi: 10.1002/mc.20436

51. Li H, Zhou F, Wang H, Lin D, Chen G, Zuo X, et al. Knockdown of EIF3D suppresses proliferation of human melanoma cells through G2/M phase arrest. Biotechnol Appl Biochem. (2015) 62:615-20. doi: 10.1002/ bab. 1305

52. Fowles JS, Denton CL, Gustafson DL. Comparative analysis of MAPK and PI3K/AKT pathway activation and inhibition in human and canine melanoma. Vet Comp Oncol. (2015) 13:288-304. doi: 10.1111/vco.12044

53. Wei BR, Michael HT, Halsey CH, Peer CJ, Adhikari A, Dwyer JE, et al. Synergistic targeted inhibition of MEK and dual PI3K/mTOR diminishes viability and inhibits tumor growth of canine melanoma underscoring its utility as a preclinical model for human mucosal melanoma. Pigment Cell Melanoma Res. (2016) 29:643-55. doi: 10.1111/pcmr.12512

54. London CA. Tyrosine kinase inhibitors in veterinary medicine. Top Companion Anim Med. (2009) 24:106-12. doi: 10.1053/j.tcam.2009.02.002

55. Helal-Neto E, Brandão-Costa RM, Saldanha-Gama R, Ribeiro-Pereira C, Midlej V, Benchimol M, et al. Priming endothelial cells with a melanomaderived extracellular matrix triggers the activation of $\alpha v \beta 3 /$ VEGFR2 axis. $J$ Cell Physiol. (2016) 231:2464-73. doi: 10.1002/jcp.25358

56. Sato A, Sunayama J, Matsuda K, Tachibana K, Sakurada K, Tomiyama A, et al. Regulation of neural stem/progenitor cell maintenance by PI3K and mTOR. Neurosci Lett. (2010) 470:115-20. doi: 10.1016/j.neulet.2009.12.067

57. Braeuer RR, Watson IR, Wu CJ, Mobley AK, Kamiya T, Shoshan E, et al. Why is melanoma so metastatic? Pigment Cell Melanoma Res. (2014) 27:19-36. doi: $10.1111 / \mathrm{pcmr} .12172$

58. Asundi J, Reed C, Arca J, McCutcheon K, Ferrando R, Clark S, et al. An antibody-drug conjugate targeting the endothelin $\mathrm{B}$ receptor for the treatment of melanoma. Clin Cancer Res. (2011) 17:965-75. doi: 10.1158/1078-0432.CCR-10-2340

59. Sandhu S, McNeil CM, LoRusso P, Patel MR, Kabbarah O, Li C, et al. Phase I study of the anti-endothelin B receptor antibody-drug conjugate DEDN6526A in patients with metastatic or unresectable cutaneous, mucosal, or uveal melanoma. Invest New Drugs. (2019) 1-11. doi: 10.1007/s10637-019-00832-1

60. Luca M, Huang S, Gershenwald JE, Singh RK, Reich R, Bar-Eli M. Expression of interleukin- 8 by human melanoma cells up-regulates MMP-2 activity and increases tumor growth and metastasis. Am J Pathol. (1997) 151:1105-13.

61. Varney ML, Johansson SL, Singh RK. Distinct expression of CXCL8 and its receptors CXCR1 and CXCR2 and their association with vessel density and aggressiveness in malignant melanoma. Am J Clin Pathol. (2006) 125:209-16. doi: 10.1309/VPL5R3JR7F1D6V03

62. Satow R, Nakamura T, Kato C, Endo M, Tamura M, Batori R, et al. ZIC5 drives melanoma aggressiveness by PDGFD-mediated activation of FAK and STAT3. Cancer Res. (2017) 77:366-77. doi: 10.1158/0008-5472.CAN-16-0991
63. Ottnod JM, Smedley RC, Walshaw R, Hauptman JG, Kiupel M, Obradovich JE. A retrospective analysis of the efficacy of Oncept vaccine for the adjunct treatment of canine oral malignant melanoma. Vet Comp Oncol. (2013) 11:219-29. doi: 10.1111/vco.12057

64. Treggiari E, Grant JP, North SM. A retrospective review of outcome and survival following surgery and adjuvant xenogeneic DNA vaccination in 32 dogs with oral malignant melanoma. J Vet Med Sci. (2016) 78:845-50. doi: 10.1292/jvms.15-0510

65. Verganti S, Berlato D, Blackwood L, Amores-Fuster I, Polton GA, Elders R, et al. Use of Oncept melanoma vaccine in 69 canine oral malignant melanomas in the UK. J Small Anim Pract. (2017) 58:10-6. doi: 10.1111/jsap.12613

66. Castelli C, Rivoltini L, Andreola G, Carrabba M, Renkvist N, Parmiani G. T-cell recognition of melanoma-associated antigens. J Cell Physiol. (2000) 182:323-31. doi: 10.1002/(SICI)1097-4652(200003) 182:3\%3C323::AID-JCP2\%3E3.0.CO;2-\%23

67. Park EJ, Kim JH, Seong RH, Kim CG, Park SD, Hong SH. Characterization of a novel mouse cDNA, ES18, involved in apoptotic cell death of T-cells. Nucleic Acids Res. (1999) 27:1524-30. doi: 10.1093/nar/27.6.1524

68. Zou JX, Revenko AS, Li LB, Gemo AT, Chen HW. ANCCA, an estrogenregulated $\mathrm{AAA}+\mathrm{ATPase}$ coactivator for $\mathrm{ER} \alpha$, is required for coregulator occupancy and chromatin modification. Proc Natl Acad Sci USA. (2007) 104:18067-72. doi: 10.1073/pnas.0705814104

69. Lee Y, Lee JK, Ahn SH, Lee J, Nam DH. WNT signaling in glioblastoma and therapeutic opportunities. Lab Investig. (2016) 96:137-50. doi: 10.1038/labinvest.2015.140

70. Song Y, Li L, Ou Y, Gao Z, Li E, Li X, et al. Identification of genomic alterations in oesophageal squamous cell cancer. Nature. (2014) 509:91-5. doi: $10.1038 /$ nature 13176

71. Arend RC, Londoño-Joshi AI, Straughn JM Jr, Buchsbaum DJ. The Wnt/Bcatenin pathway in ovarian cancer: a review. Gynecol Oncol. (2013) 131:772-9. doi: 10.1016/j.ygyno.2013.09.034

72. Howe LR, Brown AM. Wnt signaling and breast cancer. Cancer Biol Ther. (2004) 3:36-41. doi: 10.4161/cbt.3.1.561

73. Deitrick J, Pruitt WM. Wnt/ $\beta$ catenin-mediated signaling commonly altered in colorectal cancer. Prog Mol Biol Transl Sci. (2016) 144:49-68. doi: 10.1016/bs.pmbts.2016.09.010

74. Schneider JA, Logan SK. Revisiting the role of $\mathrm{Wnt} / \beta$-catenin signaling in prostate cancer. Mol Cell Endocrinol. (2018) 462:3-8. doi: 10.1016/j.mce.2017.02.008

75. Rapp J, Jaromi L, Kvell K, Miskei G, Pongracz JE. WNT signaling - lung cancer is no exception. Respir Res. (2017) 18:167. doi: 10.1186/s12931-017-0650-6

76. Kaur A, Webster MR, Weeraratna AT. In the Wnt-er of life: Wnt signalling in melanoma and ageing. Br J Cancer. (2016) 115:1273-9. doi: $10.1038 /$ bjc. 2016.332

77. Bergman PJ. Canine oral melanoma. Clin Tech Small Anim Pract. (2007) 22:55-60. doi: 10.1053/j.ctsap.2007.03.004

Conflict of Interest: The authors declare that the research was conducted in the absence of any commercial or financial relationships that could be construed as a potential conflict of interest.

Copyright (c) 2019 Brocca, Ferraresso, Zamboni, Martinez-Merlo, Ferro, Goldschmidt and Castagnaro. This is an open-access article distributed under the terms of the Creative Commons Attribution License (CC BY). The use, distribution or reproduction in other forums is permitted, provided the original author(s) and the copyright owner(s) are credited and that the original publication in this journal is cited, in accordance with accepted academic practice. No use, distribution or reproduction is permitted which does not comply with these terms. 